Revista Eletrônica de Farmácia

Eletronic Journal of Pharmacy

ISSN 1808-0804

doi $10.5216 /$ ref.v15ie.47586

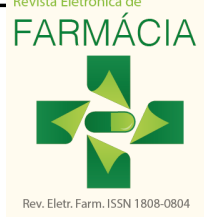

Revisão

\title{
Cromatografia contracorrente: princípios e aplicações
}

\section{Countercurrent chromatography: principles and applications}

\section{Cromatografía contracorriente: principios y aplicaciones}

\author{
BÜTTENBENDER, Sabrina Laíz ${ }^{1, *}$, SIMON, Elisa de Saldanha ${ }^{1}$, VOLPATO, and Nadia Maria ${ }^{1}$ \\ ${ }^{1}$ Programa de Pós-Graduação em Ciências Farmacêuticas da Universidade Federal do Rio Grande do Sul \\ *sbuttenbender@gmail.com
}

Resumo. Introdução: Esta revisão aborda a cromatografia contracorrente (CCC), técnica utilizada para separação em que são utilizadas duas fases líquidas imiscíveis, compostas por uma mistura de solventes. As vantagens da CCC frente a outras técnicas cromatográficas estão relacionadas principalmente à ausência de um suporte sólido. Metodologia: São apresentados os principais aspectos em relação à evolução da técnica, ao desenvolvimento metodológico e às aplicações em produtos naturais e na enantiosseparação, relatadas em estudos publicados entre janeiro de 2013 e agosto de 2016. Resultados e Discussão: A seleção do sistema de solventes bifásico (SSB) é um ponto crítico do método e tem sido alvo de importantes estudos, os quais incluem também o desenvolvimento de modelos completamente teóricos para escolha do sistema de solventes ideal. As tendências apontam para o uso de alcanos, já tradicionais em CCC, em que podem ser adicionados agentes modificadores, como ácidos, bases e seletores quirais. Algumas inovações nos modos de eluição descrevem os gradientes de polaridade, a extrusão da fase estacionária, a reciclagem de fase móvel e o refinamento por zona de $\mathrm{pH}$. Conclusões: $\mathrm{O}$ emprego da CCC para a separação de produtos de origem vegetal e de enantiômeros é destacado, evidenciando o potencial desta técnica na separação de compostos com atividade biológica.

Palavras-chave: Cromatografia. Solventes. Produtos Biológicos.

Abstract. Introduction: This review addresses the countercurrent chromatography (CCC), a separation technique that uses two immiscible liquid phases, composed of a solvents mixture. The advantages of CCC over other chromatographic techniques are mainly associated to the absence of a solid support. Methodology: The main aspects regarding the evolution of the technique, methodological development and applications to natural products and enantioseparation reported in studies published between 2013 and August 2016 are presented. Results and Discussion: The biphasic solvent system (BSS) selection is a critical point of the method, and it has been the subject of important studies, including the development of completely theoretical models to choose the ideal solvent system. Trends observed addresses to the use of alkanes, well established in CCC, in which it is possible to add some modifying agents, like acids, bases and chiral selectors. Some modifications in elution modes are reported, such as polarity gradients, stationary phase extrusion, mobile phase recycling and $\mathrm{pH}$-zone refining. Conclusions: The use of CCC for the separation of vegetal products and enantiomers is highlighted, pointing the potential of this technique for the separation of biologically active compounds.

Key-words: Chromatography. Solvents. Biological Products.

Resumen. Introducción: Esta revisión aborda la cromatografía contracorrente (CCC), una técnica de separación que utiliza das fases líquidas inmiscibles, compuestas por una mezcla de solventes. Las ventajas de la (CCC) frente a otras técnicas cromatográficas están relacionadas principalmente a la ausencia de un soporte sólido. Metodología: Se presentaron los principales aspectos en relación a la evolución de la técnica, al desarrollo metodológico y las aplicaciones en productos naturales y en la enantioseparación, reportadas en estudios publicados entre 2013 y agosto de 2016 . Resultados y Discusión: La selección del sistema de solventes bifásico (SSB) es un punto crítico del método y, ha sido objeto de importantes estudios, incluyendo también el desarrollo de modelos completamente teóricos para elegir el sistema de solventes ideal. Las tendencias apuntan al uso de alcanos, ya tradicionales en CCC, en que se pueden agregar agentes modificadores, como ácidos, bases y selectores quirales. Se describieron algunas innovaciones en los modos de elución, como gradientes de polaridad, extrusión de fase estacionaria, reciclaje de fase móvil y refinación por zona de $\mathrm{pH}$. Conclusiones: Se destacó el empleo de la CCC para la separación de productos de origen vegetal y de enantiómeros, evidenciando el potencial de esta técnica para la separación de compuestos biológicamente activos.

Palabras-clave: Cromatografía. Solventes. Productos biológicos. 


\section{Introdução}

A cromatografia contracorrente (CCC) é uma técnica de separação por meio da partição da amostra em um sistema líquido-líquido, sem envolver uma fase sólida. A fase estacionária (FE) e a fase móvel (FM) são líquidos imiscíveis, obtidas a partir de uma mistura de solventes previamente equilibrados. A proporção relativa dos solutos da amostra em cada uma das fases está relacionada aos respectivos coeficientes de partição(1).

Em comparação a outras técnicas cromatográficas, as principais vantagens da CCC estão relacionadas à ausência de suporte sólido para FE, permitindo a recuperação total da amostra injetada, visto que não há adsorção de analitos, processo irreversível que pode levar ao esgotamento do suporte e à perda de amostra(2). Ainda, o consumo de solvente por carga de amostra processada é menor em CCC, quando comparado com a cromatografia líquida de alta eficiência (CLAE), devido à natureza líquida da FE. A razão entre o volume de FE disponível para separação e o volume de FM é em torno de $80 \%$ na coluna de CCC, em contraste a $20 \%$ da CLAE, onde a fração da coluna que participa ativamente do processo de separação é limitada pela interface com a $\mathrm{FM}(1,3)$.

Em CCC, a eficiência do processo está relacionada à retenção da FE na coluna. A variação no volume relativo de FE na coluna é capaz de modificar a resolução entre compostos com diferentes coeficientes de partição, melhorando a eficiência de uma separação específica(4). Dispondo apenas de alguns solventes de uso comum, pode-se ter grande variedade de sistemas de solventes, apenas variando as proporções de cada solvente no sistema(5).

Apesar das vantagens relacionadas ao uso de FE líquida e do crescente número de estudos relacionados à separação de compostos naturais, enantiômeros, macromoléculas e impurezas, ainda há pouca aplicação da CCC na indústria farmoquímica e farmacêutica. Ảinda assim, a mesma é uma técnica com uso industrial promissor, pois o escalonamento de um método analítico piloto pode ser feito de forma rápida e previsível, com elevadas taxas de pureza e rendimento $(4,6)$, sendo este fato um diferencial em comparação com outras técnicas cromatográficas.

Este estudo teve como objetivo revisar a evolução da técnica de CCC, abordando aspectos relacionados aos sistemas de solventes e modos de eluição, enfatizando os estudos de separação de produtos naturais e de enantiômeros publicados recentemente.

\section{Metodologia}

Para o desenvolvimento desta revisão integrativa, foi realizada uma pesquisa bibliográfica nas bases de dados Scopus e Web of Science, utilizando os termos de busca "countercurrent chromatography" e "counter-current chromatography". Para a construção de indicadores relacionados às publicações de aplicação prática da técnica, utilizou-se a base de dados Scopus, limitando apenas para resultados em inglês, publicados entre janeiro de 2013 e agosto de 2016. A busca retornou 428 resultados, sendo que aqueles artigos cujo acesso ao texto completo não estava disponível foram excluídos. Os trabalhos encontrados contendo aplicações práticas de CCC de alta velocidade e de alta eficiência foram compilados em uma planilha eletrônica, descrevendo as informações: fonte da publicação, tipo de equipamento utilizado, escala de trabalho e área de aplicação. Para o embasamento teórico da presente revisão foram também utilizados artigos científicos, de revisão e capítulos de livros anteriores a este período. Trabalhos cujo enfoque principal era aprimoramento instrumental e modelagem matemática em CCC não foram exaustivamente analisados, sendo segregados.

\section{Resultados e discussão \\ Breve Evolução da CCC}

Em 1949, com o conceito de distribuição contracorrente, foi desenvolvido o equipamento de Craig e Post, que consistia numa série de tubos de ensaio contendo a FE e uma vazão de FM que os atravessava, sendo que a amostra era colocada no primeiro tubo. Este equipamento não é mais utilizado, devido à necessidade de grande espaço físico e a sua fragilidade. Na distribuição contracorrente, o equilíbrio é completado antes da transferência de fase $(7,8)$.

No final da década de 1960 foram desenvolvidos os primeiros protótipos e estudos envolvendo o princípio da CCC, por Yoichiro Ito(2). Debates sobre a nomenclatura da técnica têm a acompanhado desde o início do seu desenvolvimento. O próprio nome gera controvérsias, uma vez que as duas fases não realizam uma circulação contracorrente entre si: apenas um dos líquidos flui enquanto o outro está retido no sistema, através de um campo de força gravitacional gerado por um movimento centrífugo. Porém, a nomenclatura envolvida foi adotada por seus usuários desde seu desenvolvimento e, em 2009, a IUPAC publicou um relatório técnico intitulado Countercurrent Chromatography in Analytical Chemistry, no qual aborda as definições e a terminologia geral relacionada à $\operatorname{CCC}(3,9,10)$.

Desde o início dos anos 1970, quando Ito e Bowman(11) chamaram de Cromatografia Contracorrente a técnica cromatográfica que desenvolveram usando um tubo helicoidal preenchido com duas fases líquidas imiscíveis, sem a presença de um suporte sólido, a CCC vem sendo constantemente aprimorada por meio de diferentes princípios e equipamentos.

Um sistema cromatográfico preparado para executar CCC consiste de uma bomba que promove a vazão de FM, um injetor, um sistema tubular espiral, chamado de coluna, que realiza um movimento planetário e origina um campo de força gravitacional que mantem a FE retida, um coletor de frações e um detector(3). As colunas utilizadas 
em CCC foram classificadas em duas principais categorias, considerando a natureza, constante ou não, do campo de força gravitacional gerado pelo instrumento: as colunas hidrostáticas e as colunas hidrodinâmicas $(2,3,9,12)$. Em equipamentos hidrostáticos, o campo de força gravitacional é derivado da rotação em torno de um único eixo. Nos equipamentos hidrodinâmicos, o campo de força gravitacional gerado na coluna tubular espiralada é variável, devido à força gravitacional resultante da combinação dos movimentos síncronos de centrifugação e de rotação em torno do próprio eixo do suporte em que a coluna está envolta. Este movimento planetário é chamado de "tipo J" $(10,13)$.

Desde o surgimento da CCC foram desenvolvidas algumas variações técnicas, utilizando o sistema hidrostático ou hidrodinâmico. Como exemplo de sistemas hidrostáticos temos a cromatografia contracorrente de gotículas (DCCC, do inglês droplet countercurrent chromatography) e a cromatografia de rotação locular (RLCCC, do inglês rotation locular countercurrent chromatography) $(2,13)$.

Visando melhorar a resolução entre os sinais cromatográficos, diversos arranjos geométricos de colunas e eixos de rotação foram desenvolvidos(13), promovendo também aumento no volume de FE retida na coluna. O sistema chamado "J" é utilizado atualmente em equipamentos de cromatografia contracorrente de alta velocidade (HSCCC, do inglês high-speed countercurrent chromatography) e cromatografia contracorrente de alta eficiência (HPCCC, do inglês high-performance countercurrent chromatography).

Os equipamentos de HSCCC operam a velocidades de rotação que geram forças de aceleração de até 80 vezes a aceleração da gravidade da Terra $(80 \mathrm{~g})$. De maneira geral, as análises realizadas nestes instrumentos têm duração mínima de 120 minutos(14).

Na década de 2000, desenvolveu-se a técnica de HPCCC, em que equipamentos com o mesmo mecanismo de funcionamento foram capazes de atingir maiores velocidades $(1600 \mathrm{rpm})$, gerando forças gravitacionais de $240 \mathrm{~g}$ no interior da coluna(15). Separações por HPCCC tem menor tempo de corrida, na ordem de dezenas de minutos, mantendo boa resolução de análise em escala analítica e preparativa, com maior retenção de $\mathrm{FE}(14)$.

Guzlek, Wood e Janaway(15) publicaram um trabalho comparando equipamentos de HSCCC e HPCCC, concluindo que campos de força gravitacionais de maior intensidade retêm maior quantidade de FE na coluna, levando a separações com menor tempo de corrida. Ainda evidenciaram que os métodos em HPCCC foram capazes de processar uma quantidade dez vezes maior de amostra, com maior resolução entre os sinais cromatográficos(15).

Outro trabalho confrontando diferentes instrumentos de CCC foi realizado por Zhang e colaboradores(16), que apresentaram uma análise comparativa de custo-eficiência. Em comparação com métodos desenvolvidos em HSCCC publicados anteriormente, o método em HPCCC teve melhor desempenho, com maiores quantidades de amostra bruta

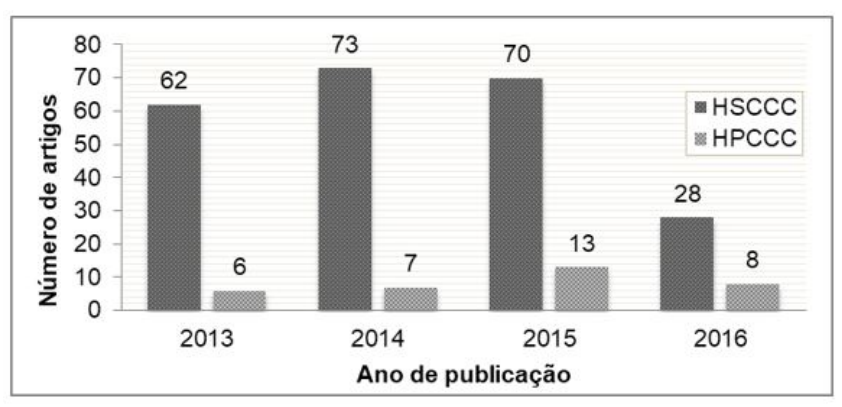

Fonte: Elaborado pelos autores.

Figura 1. Artigos publicados com estudos de HSCCC e HPCCC.

processada e de composto isolado recuperado.

Entretanto, ainda há poucos instrumentos de HPCCC disponíveis e a predominância de publicações envolvendo HSCCC é marcante (FIGURA 1). O desenvolvimento técnico da CCC ainda está em evolução e, dentre as publicações levantadas na base de dados, aproximadamente $13 \%$ eram estudos relacionados a novas metodologias ou modificações instrumentais, estas últimas envolvendo principalmente a geometria da coluna de separação(17).

\section{Princípio Fundamental da CCC}

O princípio de separação em CCC é baseado na partição de um soluto entre duas fases líquidas imiscíveis, uma sendo mantida estacionária no interior da coluna, e outra móvel, no processo cromatográfico dentro da coluna. Em equipamentos hidrodinâmicos, a FE é mantida no interior da coluna devido ao campo de força gravitacional gerado no equipamento, resultante da combinação dos movimentos síncronos de rotação e revolução. A FIGURA 2A ilustra um instrumento que realiza este movimento planetário, conhecido como "tipo J", em que os eixos de rotação e revolução são paralelos. A estrutura na qual a coluna está enrolada gira em torno do seu próprio eixo e em torno do eixo central do equipamento na mesma velocidade angular () e na mesma direção(2).

Este movimento mantém a fase menos densa do sistema em uma das extremidades da coluna, chamada de cabeça, enquanto a fase mais densa tende a se movimentar em direção à outra extremidade, chamada de cauda. Esta tendência permite a operação do equipamento em dois modos. No modo normal, a coluna é inicialmente preenchida pela fase mais densa (a fase inferior do sistema de solventes) e, então, a FM de menor densidade (a fase superior) é bombeada da cauda para a cabeça da coluna. Já no modo reverso, a coluna é preenchida com a fase menos densa e a fase mais densa é bombeada da cabeça para a cauda. Desta forma, com velocidade de rotação e vazão adequadas, é possível reter mais de $50 \%$ de FE na coluna, que é o percentual mínimo indicado para separações eficientes $(3,18)$.

Este comportamento proporciona, ainda, um eficiente contato do soluto com as duas fases líquidas. Na FIGURA 


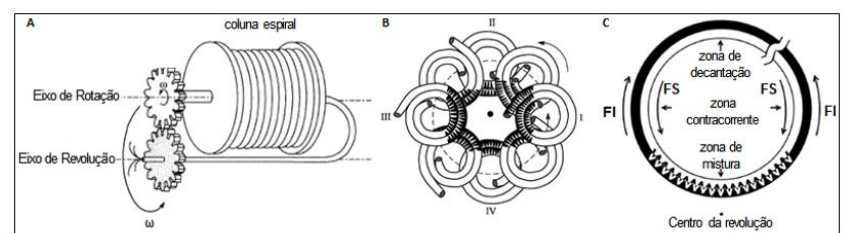

Fonte: Adaptado de $\operatorname{Ito}^{(2)}$ e Ito ${ }^{(10)}$.

Figura 2. (A) Coluna espiral de equilíbrio hidrodinâmico que realiza movimento planetário síncrono "tipo J"; (B) Distribuição das fases no interior da coluna espiral em diferentes momentos do movimento (I, II, III e IV); (C) Distribuição das fases em uma volta da coluna espiral. FI: Fase Inferior; FS: Fase Superior.

2B é possível observar um esquema com várias posições (I, II, III e IV) que a coluna atinge durante o movimento planetário. O interior da coluna é dividido em zonas diferentes, no que se refere a força gravitacional resultante (FIGURA 2C). Próximo ao eixo central de rotação ocorre a mistura das duas fases líquidas, e na região restante tem-se a zona de decantação, em que o campo de força gravitacional é alto(3). Desse modo, o campo de força gravitacional produzido no interior da coluna é alternante e não constante. Estabelece-se um equilíbrio hidrodinâmico entre as fases, sendo que cada uma delas pode ocupar, aproximadamente, metade do volume total da coluna(18).

Em CCC, o coeficiente de partição $(\mathrm{K})$ é calculado como a razão entre a concentração de soluto na FE por aquela na FM. A faixa mais indicada de valores de Ké de 0,4 a 2,5(19). Valores baixos indicam afinidade do soluto pela FM, o que pode levar à eluição junto ao front de solvente, enquanto valores elevados estão associados à maior interação com a FE, levando a tempos de corrida mais longos(19).

\section{Sistemas de Solventes}

A maior parte dos sistemas de solventes utilizados em CCC são misturas ternárias ou quaternárias contendo água e solventes orgânicos, que se separam em duas fases, a superior e a inferior. Inicialmente, utilizou-se clorofórmio como principal solvente hidrofóbico, mas devido aos efeitos ambientais e biológicos deletérios associados a solventes clorados, popularizou-se o uso de sistemas contendo alcanos como solvente majoritário da fase superior. Sistemas que contém heptano ou hexano, acetato de etila, metanol e água em diferentes proporções são eficazes na separação de substâncias em uma ampla faixa de polaridade, abrangendo compostos lipofílicos até moderadamente polares(20). Composições pré-definidas desses solventes são propostas e tabeladas, sendo uma delas conhecida como sistema Arizona, com nomenclatura de A a Z para as diferentes proporções (FIGURA 3). Em geral, esses sistemas se separam em duas fases rapidamente, o que está relacionado a processos com boa retenção de FE (2).

Skalicka-Woźniak e Garrard(22) realizaram um levantamento dos sistemas de solventes bifásicos utilizados para purificação de produtos naturais por CCC e CPC no período de 1984 a 2014. Os quatro solventes mais listados

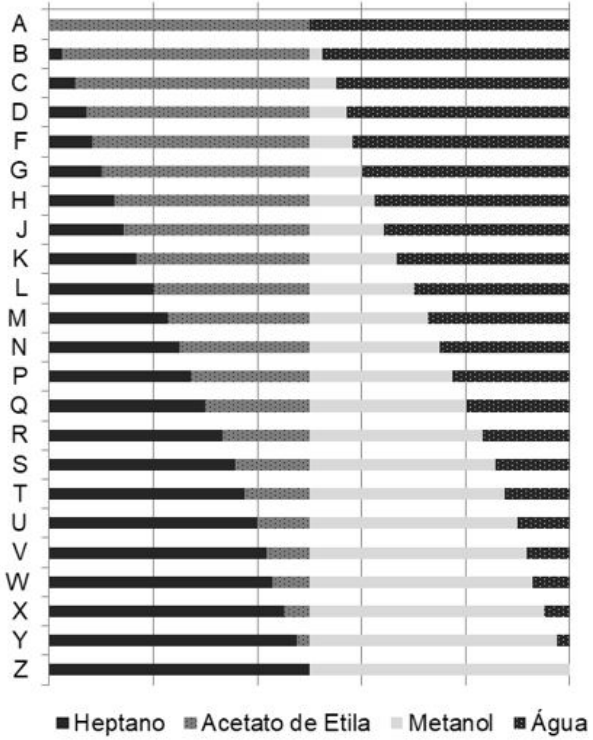

Fonte: Adaptado de Berthod, Hassoun e Ruiz-Angel(21).

Figura 3. Composição percentual (v/v/v/v) das 23 misturas nomeadas de A a Z.

nas composições foram água, acetato de etila, metanol e hexano, evidenciando a popularidade dessa família de solventes, conhecida como HEMWat (do inglês hexane/ethyl acetate/methanol/water). A separação de amostras de caráter hidrofílico é direcionada ao uso de menor proporção de alcano, ou então de outros solventes, como as combinações entre clorofórmio, metanol e água (ChMWat) ou hexano, butanol e água. Para o isolamento de compostos ionizáveis, é possível ajustar o valor de $\mathrm{K}$ pelo controle do $\mathrm{pH}$, utilizando ao invés de água, uma solução aquosa de sulfato de amônia ou ácido trifluoracético, por exemplo(23,24).

Estudos recentes têm utilizado sistemas bifásicos aquosos, principalmente para separação de compostos bioativos, visto que preservam a atividade e a conformação das substâncias em solução(25,26). A separação do sistema aquoso em duas fases pode ser promovida por duas estratégias. A primeira, e mais amplamente aplicada, é a utilização de polímeros, como polietilenoglicol de alto peso molecular, que confere característica menos polar a uma das fases(27). A segunda é pela adição de líquidos iônicos, que são sais líquidos de viscosidade elevada em temperatura ambiente compostos, por exemplo, pelo cátion imidazólio combinado a diferentes ânions(28).

\section{Seleção do sistema de solventes bifásico (SSB)}

Em CCC, a etapa crítica em estudos almejando o isolamento de uma substância é a seleção do sistema de solventes bifásico (SSB). Diferente de outras metodologias de separação, a FE e a FM não são independentes, pelo contrário, a composição de cada uma das fases está relacionada ao 
equilíbrio formado entres ambas em um sistema de diferentes solventes. Por esta razão, não é possível escolher a FE separadamente da FM. São escolhidas em uma mesma etapa, em que é avaliada a natureza e a proporção de cada componente da mistura de solventes que formará as duas fases.

A seleção empírica do SSB geralmente envolve processos iterativos de determinação dos valores de $\mathrm{K}$ em experimentos de partição ou dos fatores de retenção (Rf) em cromatografia em camada delgada (CCD)(29). Como a possibilidade de combinações de solventes é praticamente infinita, alguns protocolos foram montados para auxiliar na busca sistemática de um sistema de solventes adequado(23).

Para selecionar o sistema de solventes por CCD, uma porção de amostra é dissolvida em SSB com mesmo volume de fase inferior e superior. Alíquotas iguais coletadas de ambas as fases são aplicadas em uma placa cromatográfica de sílica e eluídas com a fase menos polar, avaliando-se então os valores de Rf dos componentes a serem separados. Utilizando diferentes proporções de solventes HEMWat e ChMWat, Friesen e Pauli(19) demonstraram a relação existente entre os valores de Rf e os coeficientes de partição obtidos experimentalmente. É desejável que os valores de Rf estejam entre 0,29 e 0,71 , pois estão relacionados a valores de K entre 0,4 e 2,5. No entanto, esta correspondência não necessariamente implica um bom resultado em CCC, já que o mecanismo de separação por CCD envolve também, além da partição, a adsorção da amostra no suporte sólido(1).

Para os experimentos de partição, a amostra em estudo é dissolvida nas proporções de solventes em teste. Após o equilíbrio, é feita a quantificação do analito em cada uma das fases, geralmente por CLAE, e a partir desses resultados é calculado o valor de K para um determinado SSB. Obtendo-se o valor de $\mathrm{K}$ do analito em equilíbrio estático é possível inferir com boa correlação o valor de $\mathrm{K}$ no equilíbrio hidrodinâmico de CCC(30), o que justifica o amplo uso dessa estratégia em grande parte dos estudos(29).

A predição ou estimativa do valor de $\mathrm{K}$ por modelos matemáticos tem sido estudada para facilitar a escolha do SSB ideal. A estimativa é feita com base nas propriedades termodinâmicas do analito e dos solventes. Ren e colaboradores(31) empregaram um método semiempírico para seleção do SSB para separação de sete componentes de Spirodela polyrrhiza (L.) com diferentes polaridades. A estratégia computacional utilizada no trabalho, NRTL-SAC (do inglês nonrandom two-liquid segment activity coefficient), requer o resultado de três a cinco experimentos de partição para predizer o valor de K em diferentes proporções de SSB, mesmo que em famílias de solventes distintas(32). Enquanto as características termodinâmicas dos solventes utilizados podem ser extraídas da literatura, os valores experimentais de K são necessários para determinação dos parâmetros moleculares dos solutos. Com esses dados, a estrutura dos compostos alvo não precisa ser informada, o que é interessante quando se deseja separar produtos naturais cuja estrutura molecular ainda é desconhecida.

Para métodos completamente teóricos de seleção do sistema de solventes, a solubilidade de cada composto é calculada em cada uma das fases. É necessário conhecer, além da composição da fase e da estrutura molecular dos solventes, a estrutura molecular do analito, pois os modelos in silico levam em conta as características geométricas das moléculas envolvidas. O processo completo, até chegar ao cálculo de $\mathrm{K}$, requer pelo menos três plataformas de softwares diferentes(30), o que limita a aplicação de apenas estratégias computacionais para escolha do SSB.

\section{Modos de Eluição}

As aplicações mais convencionais de CCC utilizam um sistema de solventes em modo de eluição isocrático para separação de componentes majoritários de misturas complexas(9). Diversas alternativas têm sido empregadas visando o isolamento de compostos de diferentes polaridades ou corridas de menor duração, como, por exemplo, a eluição em gradiente de polaridade.

Alterar a proporção dos solventes da FM durante a corrida de CCC foi uma das primeiras estratégias empregadas para separar componentes com uma ampla faixa de valores de $\mathrm{K}$ de uma mistura complexa. Para evitar a perda de FE durante este processo, o ideal é que o sistema de solventes seja robusto, mantendo a composição da FE estável mesmo quando a composição da outra fase é alterada(33). O gradiente pode ser linear ou não, sendo este último o mais empregado, no qual em algum ponto da corrida com o sistema de solventes primário é bombeada uma nova $\mathrm{FM}(34)$. Tipicamente, em uma corrida em modo reverso, a nova FM é composta por uma proporção menos polar dos mesmos solventes utilizados no SSB original, para eluir os analitos de menor polaridade.

Yang e colaboradores(35) isolaram quatro podofilotoxinas com modos de eluição isocrático e gradiente, utilizando HEMWat (4:6:3:7 e 4:6:4:6, v/v/v/v). Os testes foram realizados em HSCCC em modo reverso, variando a composição da FM constituída por metanol e água. Os modos de gradiente linear e não-linear apresentaram melhor resolução. Ainda, o gradiente não-linear apresentou menor tempo de corrida (360 minutos) em relação ao linear (420 minutos).

Para otimizar a separação de compostos com valores de $\mathrm{K}$ muito diferentes também pode ser usado um gradiente de fluxo. O aumento da vazão de FM durante a corrida permite diminuir o tempo de separação para compostos com afinidade pela FE(34). Entretanto, a resolução pode ser afetada, devido à maior perda de FE causada por fluxos mais eleva$\operatorname{dos}(36)$.

Todavia, Ko e colaboradores(37) conseguiram melhor resolução e separação aplicando um gradiente de fluxo. Foram testados diferentes fluxos para a separação por HPCCC de cinco oligostilbenos, após otimização do SSB HEMWat $(4: 8: 4: 10, \mathrm{v} / \mathrm{v} / \mathrm{v} / \mathrm{v})$ utilizado em modo reverso. Foi testado 
um gradiente, onde um fluxo de $4 \mathrm{~mL} / \mathrm{min}$ foi mantido nos 70 minutos iniciais, quando então passou para $8 \mathrm{~mL} / \mathrm{min}$. A alta velocidade atingida pelo equipamento de HPCCC foi capaz de reter $65 \%$ da FE, mesmo com alta vazão de FM.

Já para compostos ionizáveis, que quando em sua forma neutra possuem valores de $\mathrm{K}$ muito próximos, pode ser aplicado um gradiente de $\mathrm{pH}$. Durante a eluição, são bombeadas fases móveis aquosas ajustadas a diferentes valores de $\mathrm{pH}$, de acordo com o pKa dos ácidos ou bases que se deseja separar(29,38).

Na década de 1990 desenvolveu-se a técnica de CCC com refinamento por zona de $\mathrm{pH}$, a qual separa compostos ionizáveis utilizando modificadores de natureza ácida e básica no sistema de solventes. Diferente do modo de eluição em gradiente de $\mathrm{pH}$, em que a adição de base ou ácido é feita somente na $\mathrm{FM}(34)$, no refinamento por zona de $\mathrm{pH}$ há adição de baixas concentrações de modificadores de $\mathrm{pH}$ em ambas as fases do sistema.

Nesta técnica, pela combinação da adição de um agente de retenção na FE e de um agente de eluição na FM é possível reter os analitos e fazer uma eluição modulada de acordo com suas diferenças de polaridade e pKa(3). É uma estratégia para otimização de separação que tem sido utilizada em instrumentos convencionais de CCC em escala preparativa, principalmente para separação de alcaloides, ácidos orgânicos, aminoácidos e peptídeos $(34,39)$. Inicialmente, a coluna é preenchida com a FE contendo o agente de retenção. Após a injeção da amostra, sob velocidade de rotação desejada, é bombeada FM contendo o agente de eluição, que irá gradativamente entrar em equilíbrio com o agente de retenção, criando zonas bem definidas de diferentes valores de $\mathrm{pH}$, onde as moléculas podem estar protonadas ou não(40). Assim, analitos de diferentes valores de $\mathrm{pKa}$ irão se deslocar para a FM ou FE de acordo com a afinidade das espécies formadas.

Alguns ácidos graxos saturados e insaturados possuem mesmo K e são coeluídos em separações por CCC. Englert e Vetter(41) utilizaram o refinamento por zona de pH para separação de ácidos graxos de óleo de girassol, com SSB composto por hexano/acetonitrila/metanol/água $(4: 7: 1,4 / 0,5 \mathrm{v} / \mathrm{v} / \mathrm{v} / \mathrm{v})$. Foram adicionados $20 \mathrm{mM}$ de amônia (NH3) na fase inferior e $35 \mathrm{mM}$ de ácido trifluoroacético (TFA) na fase superior, sendo que foram testadas corridas em modo normal e reverso. No modo reverso ainda houve coeluição de alguns ácidos, mas no modo normal (NH3 como agente de retenção e TFA como agente de eluição) os analitos foram recuperados com pureza maior de $95 \%$.

Outra otimização de eluição pode ser feita com a extrusão da FE, voltada para separação de amostras complexas, em que os solutos possuem grandes diferenças de polaridade entre si. O objetivo é acelerar a eluição dos compostos retidos na $\mathrm{FE}$, ou seja, aqueles com maiores valores de $\mathrm{K}$, que levariam mais tempo para serem eluídos na FM(24). A primeira parte do processo é uma eluição convencional, na qual, após o bombeamento de um determinado volume de FM, a eluição é continuada substituindo-se a FM pela FE, que passa a ser bombeada continuamente. Esta, retida inicialmente na coluna, é eluída completamente, permitindo que todos os componentes separados sejam recuperados(42). Em estudo realizado em escala preparativa, Paek e Lim(43) separaram sete compostos naturais de raízes de Nardostachys chinensis com atividade farmacológica, cujos valores de $\mathrm{K}$ variavam de 0,49 a 4,23.

De maneira semelhante à eluição com extrusão, existe a eluição em modo duplo, em que os dois modos de eluição, normal e reverso, são utilizados, um em seguida do outro, em uma mesma análise. A fase que está sendo bombeada no primeiro modo é trocada pela outra fase, com a troca simultânea da direção do fluxo, passando a operar no outro modo(17).

A possibilidade de otimizar a eluição em CCC se dá também pela reciclagem da FM. O efluente da coluna é retornado a ela continuamente até que se consiga a separação desejada. É como se a coluna fosse expandida, o que aumenta a eficiência de separação(44). É preciso uma adaptação instrumental, para que a tubulação de saída do detector esteja conectada à tubulação de entrada da bomba de FM. A quantidade de solvente consumida não se altera, mas há um prolongamento da duração do experimento cromatográfico(34). Uma desvantagem inerente a essa técnica é o alargamento dos sinais cromatográficos. Com o aumento do número de ciclos, os picos são estendidos, até o limite em que se deve interromper a reciclagem, para evitar a sobreposição dos sinais. Por este motivo, amostras de maior complexidade requerem um tratamento prévio, de forma que se eliminem os solutos indesejados, ou se obtenham frações mais simples dos compostos alvo. Para a separação de polifenóis, Liu e colaboradores(45) fizeram uma prépurificação do extrato de Malus hupehensis (Pamp.) Rehder por HSCCC convencional. Posteriormente, duas frações em que houve coeluição de compostos de interesse foram submetidas à HSCCC com reciclagem, que foi efetiva na separação dos compostos de estrutura similar que não haviam sido resolvidos pela técnica convencional.

\section{Sistemas de Detecção}

Embora seja recomendado o monitoramento contínuo do processo de separação, não é necessário que exista um sistema de detecção acoplado ao equipamento de CCC. Uma vez que o SSB e as condições cromatográficas estejam definidos, as frações coletadas podem ser analisadas posteriormente por CLAE, CCD ou outra técnica adequada. De modo geral, os equipamentos de CCC são acoplados aos mesmos detectores utilizados em CLAE. A detecção por ultravioleta (UV)-visível de forma contínua é a de maior emprego, sendo que $87 \%$ dos cromatogramas apresentados em isolamento de produtos naturais por CCC empregam este detector(29).

Entretanto, muitas das moléculas separadas por CCC não absorvem, ou absorvem pouca energia na faixa UV. É o caso, por exemplo, de saponinas e terpenoides, que têm sido separados por CCC acoplada a detector evaporativo por espalhamento de luz (ELS, do inglês evaporative 
light scattering)(39). A evaporação do solvente em detectores ELS é uma vantagem quando há perda de FE durante a análise. Os solventes da FE podem alterar a linha de base no cromatograma gerado pela detecção on-line por UV(46).

Juntos, os cromatogramas com detecção por UV-visível e ELS representam aproximadamente $96 \%$ das separações de produtos naturais por CCC(29). Existem estudos envolvendo o monitoramento do eluente de CCC por técnicas que forneçam informações estruturais, como ressonância magnética nuclear de próton(47) e espectrometria de massas(1).

\section{Aplicações da CCC na purificação de produtos naturais e na enantiosseparação}

Pelo levantamento realizado na base de dados Scopus, foram contabilizadas 306 publicações na área entre janeiro de 2013 e agosto de 2016 em que foi possível acesso ao texto completo. Destas, 12 artigos $(3,92 \%)$ eram de revisão, $39(12,75 \%)$ envolviam o desenvolvimento de novos instrumentos ou de novos modelos teóricos para CCC, e os 255 artigos restantes $(83,33 \%)$ eram estudos a respeito de alguma aplicação prática da técnica. Entre estes, em apenas $22(8,63 \%)$ foram realizados estudos em escala analítica. A facilidade de desenvolvimento de métodos em escalas semipreparativa e preparativa é um diferencial da CCC, tornando-a muito atrativa para as áreas que visam à obtenção de maiores quantidades de compostos purificados.

Os artigos com aplicação da CCC foram categorizados de acordo com os compostos separados entre: produtos naturais, enantiômeros, impurezas, produtos biotecnológicos e outros, que incluem pesquisas em engenharia de alimentos, por exemplo. Aproximadamente $85 \%$ dos artigos aplicaram a CCC para separação de produtos naturais, com predominância daqueles de origem vegetal.

A purificação de produtos de origem natural é um processo complexo em que, por vezes, sã̃o necessárias técnicas de separação muito variadas. A CCC se destaca de outros métodos de separação devido, principalmente, às vantagens associadas à ausência de um suporte sólido, com o custo reduzido e a possibilidade de $100 \%$ de recuperação da amostra, já que não haverá perda por adsorção ao suporte. Além disso, compostos com uma ampla diversidade de polaridade podem ser purificados. Esta versatilidade é possível, pois há inúmeros sistemas de solventes capazes de compor as FE e FM, que podem ser ajustadas de acordo com a polaridade desejada.

Em trabalho de revisão que contemplou estudos em CCC e CPC publicados entre 1984 e 2014, Skalicka-Woźniak e Garrard(22) contabilizaram um total de 2594 compostos vegetais separados por CCC. Flavonoides e flavonolignanas são a classe mais popular em separações por CCC, com 695 compostos. Terpenoides e alcaloides aparecem em segundo e terceiro lugar, com 312 e 297 substâncias, respectivamente. Esta diferença sugere que talvez a CCC esteja mais adaptada para determinados produtos naturais, particularmente flavonoides e flavonolignanas.
Costa e Leitão(48) revisaram e propuseram estratégias para o isolamento de flavonoides livres e glicosilados por CCC. Por exemplo, a adição de butanol a uma proporção de SSB da família HEMWat é capaz de otimizar o particionamento de solutos polares, como os flavonoides glicosilados e alguns flavonoides livres, pois age como modificador da fase orgânica. Também sugerem o uso de SSBs compostos por acetato de etila, butanol e água, em que é possível aplicar um gradiente de polaridade pela alteração da proporção de butanol, o que teria grande impacto na polaridade da fase orgânica, e menor impacto na fase aquosa.

Em relação aos terpenoides, Song e colaboradores(35) apontam que a baixa polaridade e a ausência de absorção UV dificultam o isolamento por tecnologias de separação comuns, e, portanto, a CCC acoplada a detectores ELS tem se mostrado adequada para a separação destes compostos. Já o isolamento e purificação de alcaloides, devido à natureza alcalina das moléculas, apresentam bons resultados principalmente em CCC com refinamento por zona de $\mathrm{pH}$, em que SSBs contendo solventes clorados, alcanos ou éter metil-terc-butílico se destacam(49).

Skalicka-Woźniak e Garrard(22), em sua revisão, também avaliaram todos os SSBs utilizados para as separações dos produtos naturais. Os sistemas foram divididos em grupos hierárquicos baseados no solvente primário da fase hidrofóbica. Para a maioria das classes, os sistemas de solventes mais empregados são aqueles baseados em alcanos, como a família HEMWat. A exceção é a classe das saponinas, em que os sistemas clorados são os mais utilizados, seguidos de outros solventes, especialmente sistemas baseados em álcoois imiscíveis em água, como álcool butílico(39).

De acordo com Friesen e colaboradores(29), a CCC tem sido empregada principalmente como uma etapa de um processo mais complexo de fracionamento de produtos naturais do que como único procedimento de separação. Em um fluxo de separação, a possibilidade de trabalhar com grandes volumes de amostra bruta, minimizando tratamentos prévios e etapas de filtração e evaporação de solventes, por exemplo, pode ser vantajosa em termos econômicos e de eficiência(29). Ainda, a variedade de solventes que pode ser utilizada em CCC possibilita o acoplamento a outras técnicas de cromatografia líquida.

Mais uma aplicação da CCC é para o que tem sido chamado de "nocaute" de compostos majoritários, principalmente na avaliação da contribuição biológica de um metabólito para a atividade global de um extrato natural. Quando se deseja isolar os compostos minoritários, que estão presentes em níveis muito baixos e às vezes até por CLAE indetectáveis, é mais efetivo remover previamente os componentes principais. Desta forma, há o enriquecimento dos compostos menores, possibilitando o seu isolamento e identificação estrutural(50). Este enriquecimento também pode ser alcançado adicionando uma fração de metabólitos minoritários previamente separada para um extrato original que não foi modificado, sendo esta abordagem chamada de DESIGNER (do inglês Depletion and Enrichment of 
Select Ingredients Generating Normalized Extract Resources)(51).

Para a separação de enantiômeros, de forma similar a outras técnicas como eletroforese capilar (EC) e CLAE, em CCC é necessário utilizar um seletor quiral (SQ), que é adicionado ao SSB. Idealmente, o SQ é uma molécula com alta enantiosseletividade em relação ao racemato alvo, solúvel em apenas uma das fases do SSB, que geralmente será utilizada como estacionária, pois assim a quantidade requerida de SQ é menor(3). Os principais SQs utilizados são derivados de ciclodextrinas (CDs), em especial de -CDs, pois apresentam, para muitas estruturas, boa estereosseletividade e habilidade de reconhecimento quiral(5).

No entanto, até o momento, dificilmente são alcançados fatores de separação cromatográfica () maiores que 1,5(52), devido à dificuldade de identificar um SQ altamente enantiosseletivo para os racematos alvo, e pela menor eficiência relacionada à técnica. Huang e Di(5) afirmam que essa desvantagem inibe o desenvolvimento e as aplicações da separação quiral por CCC, que tem evoluído mais lentamente que a enantiosseparação por outros métodos. Em contrapartida, de modo positivo, o custo menor pode tornar a CCC competitiva, uma vez que o SQ adicionado ao SSB pode ser recuperado no final da corrida. Ainda, na técnica, podem ser utilizados reagentes de grau analítico, diminuindo o custo total do método. Dessa forma, a utilização da CCC em escala preparativa é apontada como atrativa para a separação de compostos quirais.

Estratégias para otimizar a separação de enantiômeros são apresentadas na tabela 1, que lista alguns dos principais artigos de CCC quiral publicados entre 2013 e 2016. A eluição com reciclagem, aplicada por Tong, Zheng e Yan(62) e descrita em outros estudos, é uma forma de aumentar a resolução dos enantiômeros, sem recorrer a um consumo extra de solvente tampouco de SQ. É possível também recorrer ao reconhecimento quiral bifásico, o qual consiste na adição de dois SQs no SSB, um lipofílico e outro hidrofílico, com o objetivo de aumentar a enantiosseletividade(5). Também a formação de complexos entre íons metálicos e seletores quirais é uma estratégia que pode ser utilizada em CCC para a enantiosseparação de compostos que possuem grupos de caráter eletronegativo em sua estrutura, como aminas, carbonilas e hidroxilas. As ligações de coordenação que ocorrem entre complexos com íon metálico são mais estereosseletivas que as outras forças intermoleculares(58), de modo que o poder de reconhecimento quiral é aumentado, o que pode levar à separação de maior resolução e pureza(5).

A CCC quiral está no início de seu desenvolvimento, e ainda são necessárias melhorias na eficiência de separação. Huang e $\mathrm{Di}(5)$ trazem os principais aspectos a serem desenvolvidos: seletores quirais com melhor seletividade; otimização dos SSBs para que não interfiram no reconhecimento do SQ; novos designs de instrumentos que permitam melhor retenção de FE e novos métodos para aumentar a resolução.
Além da separação de compostos naturais e enantiômeros, existem diversas outras aplicações da CCC encontradas na literatura, embora menos frequentes, como, por exemplo, a identificação de impurezas e a purificação de compostos inorgânicos e fármacos sintéticos(9). Entre os produtos com atividade biológica, a maioria é de origem vegetal, mas há também exemplos recentes em preparações biotecnológicas provenientes de algas(64), fungos(65) e bactérias(66).

\section{Versatilidade da CCC}

A CCC está estabelecida como uma técnica de separação preparativa e, apesar do seu surgimento na década de 1970, segue em evolução, sendo que uma parcela dos artigos publicados na área é referente ao desenvolvimento de novos instrumentos ou novos métodos de eluição. É notável que a maioria das inovações esteja relacionada à natureza líquida de ambas as fases cromatográficas, como o refinamento por zona de pH e outras otimizações do modo de eluição.

Os processos de separação por CCC podem ser muito flexíveis, pois outras modificações podem ser aplicadas além dos usuais parâmetros ajustáveis de outras técnicas de cromatografia líquida, como polaridade e vazão da FM. Algumas das estratégias mais singulares praticadas em CCC são a troca do modo de operação pelo intercâmbio entre as fases, a extrusão da FE e o refinamento por zona de $\mathrm{pH}$ pela adição de modificadores em ambas as fases do sistema.

Neste contexto, os pesquisadores devem adotar uma abordagem lógica para desenvolvimento de novos processos de separação. Os avanços relativos à determinação do SSB adequado, em especial os modelos preditivos, são fundamentais para a praticidade de utilização da técnica e a difusão do seu uso em outros campos de pesquisa.

\section{Considerações finais}

As vantagens inerentes da técnica, como a possibilidade de injeção direta de extratos brutos, tornam a CCC uma alternativa para contornar os problemas associados com adsorção na fase sólida. Além disto, a relação custo-eficiência tem se mostrado promissora, principalmente em comparação com métodos equivalentes desenvolvidos em CLAE. O tempo de análise dos métodos desenvolvidos em CCC ainda é considerado longo, tornando-se uma desvantagem frente a outras técnicas.

A separação de compostos para avaliação de seu potencial terapêutico ou toxicológico é um dos principais propósitos dos estudos em CCC, que vai de encontro aos interesses da indústria farmacêutica e farmoquímica. A obtenção de quantidades significativas dos produtos isolados, mesmo que em concentrações baixas na mistura inicial, é um desafio para as técnicas de separação já consagradas, dentre as quais a CCC é vista como vantajosa. 


\section{Agradecimentos}

Ao Conselho Nacional de Desenvolvimento Científico e Tecnológico $(\mathrm{CNPq})$, à Universidade Federal do Rio Grande do Sul (PROBIC-FAPERGS) pelo provimento das bolsas de estudo e ao Programa de Pós-Graduação em Ciências Farmacêuticas da UFRGS pela manutenção do equipamento de HPCCC.

\section{Referências}

1. Marston A; Hostettmann K. Developments in the application of counter-current chromatography to plant analysis. J. Chromatogr. A. 2006;1112(1-2):181-194.

2. Ito Y. Golden rules and pitfalls in selecting optimum conditions for high-speed counter-current chromatography. J. Chromatogr. A. 2005;1065(2):145-168.

3. Minguillón C. Countercurrent chromatography, scope and perspectives: Application to chirotechnology. Chem. Eng. Technol. 2012;35(1):35-45.

4. Sutherland IA. Recent progress on the industrial scaleup of counter-current chromatography. J. Chromatogr. A. 2007;1151(1-2):6-13.

5. Huang X, Di D. Chiral separation by countercurrent chromatography. TrAC, Trends Anal. Chem. 2015;67:128-133.

6. Wood P, Ignatova S, Janaway L, Keay D, Hawes D, Garraard I et al. Counter-current chromatography separation scaled up from an analytical column to a production column. J. Chromatogr. A. 2007;1151(1-2):25-30.

7. Hostettmann K, Marston A, Hostettmann M. Preparative Chromatography Techniques: Applications in Natural Product Isolation, 2nd ed., Germany: Springer, 1998.

(8) Sumner N. Developing counter current chromatography to meet the needs of pharmaceutical discovery. J. Chromatogr. A. 2011;1218(36):6107-6113.

9. Berthod A, Ruiz-Ángel MJ, Carda-Broch S. Countercurrent chromatography: People and applications. J. Chromatogr. A. 2009;1216(19):4206-4217.

10. Ito Y. Counter-current motion in counter-current chromatography. J. Chromatogr. A. 2014;1372:128-132.

11. Ito Y, Bowman RL. Countercurrent Chromatography: Liquid-Liquid Partition Chromatography without Solid Support. Science. 1970;167(3916):281-283.

12. Rubio N, Minguillón C. Enantioselecive Recognition in Solution: The Case of Countercurrent Chromatography. In: Berthod A, editors. Chiral Recognition in Separation Methods: Mechanisms and Applications. Heidelberg: Springer; 2010. p. 241-274.

13. Ito Y. Origin and Evolution of the Coil Planet Centrifuge: A Personal Reflection of My 40 Years of CCC Research and Development. Sep. Purif. Rev. 2005;34(2):131-154.

14. Sutherland IA, Fisher D. Role of counter-current chromatography in the modernisation of Chinese herbal medicines. J. Chromatogr. A. 2009;1216(4):740-753.

15. Guzlek H, Wood PL, Janaway L. Performance comparison using the GUESS mixture to evaluate countercurrent chromatography instruments. J. Chromatogr. A. 2009;1216(19):4181-4186.

16. Zhang M, Ignatova S, Hu P, Liang Q, Wang Y, Sutherland I et al. Cost-efficient and process-efficient separation of geniposide from Gardenia jasminoides Ellis by highperformance counter-current chromatography. Sep. Purif. Technol. 2012;89:193-198.

17. Englert M, Vetter W. Tubing modifications for countercurrent chromatography (CCC): Stationary phase retention and separation efficiency. Anal. Chim. Acta. 2015;884:114-123.

18. Ito Y. Countercurrent chromatography. J. Biochem. Biophys. Methods. 1981;5(2):105-129.

19. Friesen JB, Pauli GF. G.U.E.S.S. - A generally useful estimate of solvent systems in CCC. J. Liq. Chromatogr. Relat. Technol. 2005;28(17):2777-2806.

20. Friesen JB, Pauli GF. Rational development of solvent system families in counter-current chromatography. J. Chromatogr. A. 2007;1151(1-2):51-59.

21. Berthod A, Hassoun M, Ruiz-Angel MJ. Alkane effect in the Arizona liquid systems used in countercurrent chromatography. Anal. Bioanal. Chem. 2005;383(2):327-340.

22. Skalicka-Woźniak K, Garrard I. A comprehensive classification of solvent systems used for natural product purifications in countercurrent and centrifugal partition chromatography. Nat. Prod. Rep. 2015;32(11):1556-61.

23. Garrard I. Simple Approach to the Development of a CCC Solvent Selection Protocol Suitable for Automation. J. Liq. Chromatogr. Relat. Technol. 2005;28(12-13):1923-1935.

24. Hu R, Pan Y. Recent trends in counter-current chromatography. TrAC, Trends Anal. Chem. 2012;40:15-27.

25. Freire MG, Pereira JFB, Francisco M, Rodríguez H, Rebelo LPN, Rogers RD et al. Insight into the Interactions That Control the Phase Behaviour of New Aqueous Biphasic Systems Composed of Polyethylene Glycol Polymers and Ionic Liquids. Chem. Eur. J. 2012;18(6):1831-1839.

26. Li W, Wu T. Rapid separation of polysaccharides using a novel spiral coil column by high-speed countercurrent chromatography. J. Sep. Sci. 2016;39(8):1404-1410.

27. Zhou XY, Zhang J, Xu RP, Ma X, Zhang ZQ. Aqueous biphasic system based on low-molecular-weight polyethylene glycol for one-step separation of crude polysaccharides from Pericarpium granati using highspeed countercurrent chromatography. J. Chromatogr. A. 2014;1362:129-134.

28. Berthod A, Carda-Broch S. Use of the ionic liquid 1-butyl-3-methylimidazolium hexafluorophosphate in countercurrent chromatography. Anal. Bioanal. Chem. 2004;380(1):168-177.

29. Friesen JB, McAlpine JB, Chen SN, Pauli GF. Countercurrent Separation of Natural Products: An Update. J. Nat. Prod. 2015;78(7):1765-1796.

30. Liu Y, Friesen JB, McAlpine JB Pauli GF. Solvent System Selection Strategies in Countercurrent Separation. Planta Med. 2015;81(17):1582-1591.

31. Ren D, Han B, Xin Z, Liu W, Ma S, Liang $\mathrm{Y}$ et al. Computation-aided separation of seven components from Spirodela polyrrhiza (L.) via counter-current chromatography. Sep. Purif. Technol. 2016;165:160-165.

32. Ren DB, Yi LZ, Qin YH, Yun YH, Deng BC, Lu HM et al. Systematic and practical solvent system selection strategy based on the nonrandom two-liquid segment activity coefficient model for real-life counter-current chromatography separation. J. Chromatogr. A. 2015;1393(47-56). 
33. Ignatova S, Sumner N, Colclough N, Sutherland I. Gradient elution in counter-current chromatography: A new layout for an old path. J. Chromatogr. A. 2011;1218(36):6053-6060.

34. Huang XY, Svetlana I, Hewitson P, Di DL. An overview of recent progress in elution mode of counter current chromatography. TrAC, Trends Anal. Chem. 2016;77(214-225).

35. Yang Z, Liu X, Wang K, Cao X, Wu S. Novel linear and step-gradient counter-current chromatography for bio-guided isolation and purification of cytotoxic podophyllotoxins from Dysosma versipellis (Hance). J. Sep. Sci. 2013;36(6):1022-1028.

36. Leitão G, Costa F. Gradient Elution in Countercurrent Chromatography. Planta Med. 2015;81(17):1592-1596.

37. Ko J, Choi J, Bae SK, Kim J, Yoon KD. Separation of five oligostilbenes from Vitis amurensis by flow-rate gradient high-performance counter-current chromatography. J. Sep. Sci. 2013;36(24):3860-3865, 2013.

38. Duanmu Q, Li A, Sun A, Liu R, Li X. Semipreparative high-speed counter-current chromatography separation of alkaloids from embryo of the seed of $\mathrm{Ne}$ lumbo nucifera Gaertn by $\mathrm{pH}$-gradient elution. J. Sep. Sci. 2010;33(12):1746-1751.

39. Song H, Lin J, Zhu X, Chen Q. Developments in highspeed countercurrent chromatography and its applications in the separation of terpenoids and saponins. J. Sep. Sci. 2016;39(8):1574-1591.

40. Ito Y. pH-zone-refining counter-current chromatography: Origin, mechanism, procedure and applications J. Chromatogr. A. 2013;1271(1):71-85.

41. Englert M, Vetter W. Overcoming the equivalentchain-length rule with $\mathrm{pH}$-zone-refining countercurrent chromatography for the preparative separation of fatty acids. Anal. Bioanal. Chem. 2015;407(18):5503-5511.

42. Wu D, Cao X, Wu S. Overlapping elution-extrusion counter-current chromatography: A novel method for efficient purification of natural cytotoxic andrographolides from Andrographis paniculata. J. Chromatogr. A. 2012;1223(53-63).

43. Paek JH, Lim SS. Preparative isolation of aldose reductase inhibitory compounds from Nardostachys chinensis by elution-extrusion counter-current chromatography. Arch. Pharm. Res. 2014;37(10):1271-1279.

44. Kostanyan AE. Simple equations to simulate closed-loop recycling liquid-liquid chromatography: Ideal and non-ideal recycling models. J. Chromatogr. A. 2015;1423:71-78

45. Liu Q, Zeng H, Jiang S, Zhang L, Yang F, Chen X et al. Separation of polyphenols from leaves of Malus hupehensis (Pamp.) Rehder by off-line two-dimensional High Speed Counter-Current Chromatography combined with recycling elution mode. Food Chem. 2015;186:139-145.

46. Michel T, Destandau E, Elfakir C. New advances in countercurrent chromatography and centrifugal partition chromatography: Focus on coupling strategy. Anal. Bioanal. Chem. 2014;406(4):957-969.

47. Hammann S, Conrad J, Vetter W. Fractionation of technical octabromodiphenyl ether by countercurrent chromatography combined with gas chromatography/mass spectrometry and offline and online (1) $\mathrm{H}$ nuclear magnetic resonance spectroscopy. J. Chromatogr. A. $2015 ; 1398: 83-93$.

48. Costa FN, Leitão GG. Strategies of solvent system selection for the isolation of flavonoids by countercurrent chromatography. J. Sep. Sci. 2010;33(3):336-347.

49. Fang L, Liu Y, Yang B, Wang X, Huang L. Separation of alkaloids from herbs using high-speed counter-current chromatography. J. Sep. Sci. 2011;34(19):2545-2558.

50. Zhao H, Hu X, Chen X, Shi S, Jiang X, Liang $\mathrm{X}$ et al. Analysis and improved characterization of minor antioxidants from leaves of Malus doumeri using a combination of major constituents' knockout with highperformance liquid chromatography-diode array detectorquadrupole time-of-flight tandem mass spectrometry. J. Chromatogr. A. 2015;1398:57-65.

51. Alvarenga RFR, Friesen JB, Nikolić D, Simmler C, Napolitano JG, Breemen R et al. K-Targeted Metabolomic Analysis Extends Chemical Subtraction to DESIGNER Extracts: Selective Depletion of Extracts of Hops (Humulus lupulus). J. Nat. Prod. 2014;77(12):2595-2604.

52. Han $\mathrm{C}, \mathrm{Xu}$ J, Wang $\mathrm{X}, \mathrm{Xu} \mathrm{X}$, Luo J, Kong L. Enantioseparation of racemic trans--viniferin using high speed counter-current chromatography based on induced circular dichroism technology. J. Chromatogr. A. 2014;1324:164-170.

53. Tong S, Wang X, Lu M, Xiong Q, Wang Q, Yan J. Enantioseparation of 2-(substituted phenyl)propanoic acids by high-speed countercurrent chromatography and investigation of the influence of substituents in enantiorecognition. J. Sep. Sci. 2016;39(8):1567-1573.

54. Zhang $\mathrm{P}$, Xie $\mathrm{X}$, Tang $\mathrm{K}$, Xu W. Chiral separation of brompheniramine enantiomers by recycling high-speed countercurrent chromatography using carboxymethyl--cyclodextrin as a chiral selector. J. Sep. Sci. 2016;39(12):2300-2306.

55. Tong S, Zhang H, Cheng D. Preparative Enantioseparation of -Substituted-2-Phenylpropionic Acids by Countercurrent Chromatography With Substituted -Cyclodextrin as Chiral Selectors. Chirality. 2015;27(11):795-801.

56. Han C, Luo J, Xu J, Zhang Y, Zhao Y, Xu X et al. Enantioseparation of aromatic -hydroxycarboxylic acids: The application of a dinuclear $\mathrm{Cu} 2$ (II)--cyclodextrin complex as a chiral selector in high speed counter-current chromatography compared with native -cyclodextrin. J. Chromatogr. A. 2015;1375:82-91.

57. Sun G, Tang K, Zhang P, Yang W, Sui G. Separation of phenylsuccinic acid enantiomers using biphasic chiral recognition high-speed countercurrent chromatography. J. Sep. Sci. 2014;37(14):1736-1741.

58. Zhang $\mathrm{P}$, Sun G, Tang K, Yang W, Sui G, Zhou C. Enantiomeric separation of oxybutynin by recycling high-speed counter-current chromatography with hydroxypropyl--cyclodextrin as chiral selector. J. Sep. Sci. 2014;37:3443-3450.

59. Tong S, Shen M, Cheng D, Zhang Y, Ito Y, Yan J. Chiral ligand exchange high-speed countercurrent chromatography: Mechanism and application in enantioseparation of aromatic -hydroxyl acids. J. Chromatogr. A. 2014;1360:110-118. 
60. Tong S, Ito Y, Ma Y. Enantioseparation of DLtryptophan by spiral tube assembly counter-current chromatography and evaluation of mass transfer rate for enantiomers. J. Chromatogr. A. 2014;1374:77-84.

61. Tong S, Shen M, Zheng Y, Chu C, Li XN, Yan J. Preparative enantioseparation of propafenone by countercurrent chromatography using di-n-butyl L-tartrate combined with boric acid as the chiral selector. J. Sep. Sci. 2013;36:3101-3106.

62. Tong S, Zheng Y, Yan J. Application and comparison of high performance liquid chromatography and high speed counter-current chromatography in enantioseparation of ()-2-phenylpropionic acid. J. Chromatogr. A. 2013;1281:79-86.

63. Tong S, Zheng Y, Yan J. Enantioseparation of chiral aromatic acids by multiple dual mode counter-current chromatography using hydroxypropyl--cyclodextrin as chiral selector. J. Sep. Sci. 2013;36(12):2035-2042.
64. Hammann S, Tillmann U, Schröder M, Vetter W. Profiling the fatty acids from a strain of the microalgae Alexandrium tamarense by means of high-speed counter-current chromatography and gas chromatography coupled with mass spectrometry. J. Chromatogr. A. 2013;1312:93-103.

65. Lu R, Liu X, Gao S, Zhang W, Peng F, Hu F et al. New tyrosinase inhibitors from paecilomyces gunnii. J. Agric. Food Chem. 2014;62(49):11917-11923.

66. He S, Wang H, Yan X, Zhu P, Chen J, Yang R. Preparative isolation and purification of macrolactin antibiotics from marine bacterium Bacillus amyloliquefaciens using high-speed counter-current chromatography in stepwise elution mode. J. Chromatogr. A. 2013;1272:15-19. 